Cuad. Soc. Esp. Cienc. For. 46(1): 245-260 (2020)

Doi: https://doi.org/10.31167/csecfv5i46.19908

\title{
El viaje de la vecería. Efectos transgeneracionales en la progenie de Pinus pinaster
}

The journey of mast seeding. Transgenerational effects on Pinus pinaster progeny

\author{
Mallo, Nerea ${ }^{2}$; Zas, Rafael ${ }^{1}$; Rodríguez, Asier ${ }^{1}$ \\ ${ }^{1}$ Departamento de Ecología Forestal. CSIC. A Carballeira, 8, 36143 Salcedo, Pontevedra \\ ${ }^{2}$ Escola de Enxeñaría Forestal Universidade de Vigo. \\ Campus Universitario da Xunqueira, A Xunqueira, S/N, 36005 Pontevedra.
}




\title{
Resumen
}

La vecería es el fenómeno por el cual ciertas especies de árboles alternan años de fuerte producción de flores, frutos o semillas con años de poca o ninguna. Un aspecto principal es que se trata de un fenómeno poblacional causado por la floración y fructificación sincronizadas de todos o la mayor parte de los individuos de una masa forestal. La alternancia entre cosechas abundantes y otras que no tiene una gran repercusión ecológica y económica tanto en el ámbito de la agricultura como en el forestal. Desde el punto de vista ecológico, la vecería repercute de forma directa en toda la comunidad de animales que dependen directa o indirectamente de las semillas o frutos, así como en la propia dinámica reproductiva de las masas arbóreas, cuya regeneración dependerá del banco de semillas disponibles en cada momento. La transmisión de caracteres a la progenie no sólo depende de la componente genética y ambiental sino también de la componente ambiental del entorno materno (plasticidad transgeneracional). Podemos entender la vecería como un fenómeno adaptativo poblacional que afecta a las propiedades de las semillas a través de mecanismos no ligados a conflictos por los recursos.

Palabras clave: vecería, sincronismo, dinámica reproductiva, efectos maternos, plasticidad transgeneracional.

\begin{abstract}
Mast seeding is the phenomenon whereby certain tree species alternate years of strong production of flowers, fruit or seeds with years of little or no production. One main aspect is that it is a population phenomenon caused by the synchronized flowering and fruiting of all or most individuals in a forest system. The alternation between abundant harvests and others that are not, has a great ecological and economic impact both in the field of agriculture and forestry. From the ecological point of view, the vegetation has a direct impact on the whole community of animals that depend directly or indirectly on the seeds or fruits, as well as on the reproductive dynamics of forest systems, whose regeneration will depend on the seed bank available at any given time. The transmission of characteristics to the progeny depends not only on the genetic and environmental component but also on the environmental component of the maternal environment (transgenerational plasticity). We can understand mast seeding as an adaptive population phenomenon that affects the properties of the seeds through mechanisms not linked to conflicts over resources.
\end{abstract}

Keywords: mast seeding, synchronism, reproductive dynamics, maternal effects, transgenerational plasticity.

\section{Resumo}

A vecería é o fenómeno polo cal certas especies de árbores alternan anos de forte produción de flores, froitos ou sementes con anos de pouca ou ningunha. Un aspecto principal é que se trata dun fenómeno poboacional causado pola floración e frutificación sincronizadas de todos ou a maior parte dos individuos dunha masa forestal. A alternancia entre colleitas abundantes e outras que non ten unha gran repercusión ecolóxica e económica tanto no ámbito da agricultura como no forestal. Desde o punto de vista ecolóxico, a vecería repercute de forma directa en toda a comunidade de animais que dependen directa ou indirectamente das sementes ou froitos, así como na propia dinámica reprodutiva das masas arbóreas, cuxa rexeneración dependerá do banco de sementes dispoñibeis en cada momento. A transmisión de caracteres á proxenie non só depende da compoñente xenética e ambiental senón tamén da compoñente ambiental da contorna materna (plasticidade transxeneracional). Podemos entender a vecería como un fenómeno adaptativo poboacional que afecta ás propiedades das sementes a través de mecanismos non ligados a conflitos polos recursos.

Keywords: vecería, sincronismo, dinámica reprodutiva, efectos maternos, plasticidade transxeneracional. 


\section{Introducción}

Todos los seres vivos enfrentan el enorme desafío de sobrevivir y reproducirse. Para las plantas, es uno de los momentos más importantes en su ciclo de vida ya que requieren de energía para reproducirse y cada estrategia tiene un coste energético diferencial. La inversión de recursos energéticos que un individuo destina a la reproducción se conoce como esfuerzo reproductivo (Avedaño, 2016). Este esfuerzo está relacionado con la elaboración de estructuras como flores, polen o frutos, así como con la elaboración de compuestos químicos volátiles que tienen la función de atraer polinizadores. En las plantas, los mecanismos reproductivos y la producción de semillas compiten constantemente por los recursos con otras funciones fisiológicas como el crecimiento y las defensas (Santos del Blanco et al., 2014).

Para las especies iteróparas, es decir, especies con varios ciclos reproductivos a lo largo de su vida, el esfuerzo reproductor puede variar a lo largo de los años. Esta variación da lugar a lo que conocemos cómo dinámica reproductiva.

En ciertas especies longevas, como los árboles, la variación en el esfuerzo reproductor a lo largo de los años es especialmente elevada, con años de mínima producción hasta años en el que ésta se dispara, un fenómeno conocido como "vecería". La vecería envuelve tres conceptos intrínsecos: variabilidad, sincronía y periodicidad. No parece un fenómeno pasivo de plasticidad a la variación ambiental interanual, sino que parece una estrategia vital con alguna finalidad biológica. Puede tener importantes consecuencias ecológicas en el funcionamiento de los ecosistemas ya que no solo tiene un efecto directo sobre la dinámica poblacional de la propia especie arbórea, sino también sobre el comportamiento de la siguiente generación.

La cantidad y calidad de las semillas, son caracteres determinados por la planta reproductora, incluyendo la constitución genética, el número de ciclos reproductivos, las condiciones ambientales y la eficiencia en el uso de los recursos destinados a la reproducción, entre otros. Es esperable que en años de máxima producción, los recursos disponibles para cada semilla sean menores, dando lugar a un compromiso entre cantidad y calidad de la producción de semillas (Sadras, 2007).

Las semillas constituyen el enlace de la transmisión entre generaciones. Además del inevitable efecto del ambiente, el éxito de la siguiente generación dependerá tanto de efectos genéticos como de efectos no genéticos de la planta madre, ambos relacionados con las características de la semilla. En este sentido, una de las características más relevantes de las semillas es su tamaño, ya que este es indicativo de los recursos disponibles para las primeras etapas de desarrollo de las plántulas tras la emergencia.

Si las ventajas son grandes, la presión selectiva ejercida por el ambiente puede favorecer a la evolución del peso de la semilla hacia tamaños más efectivos (Ramírez-Valiente et al., 2009). El peso de la semilla, por tanto, es un importante carácter de historia vital con fuertes efectos sobre el fenotipo de la descendencia, así como sobre la eficacia biológica de esta (Zas y Sampedro, 2015). 
Son muchos los factores que pueden contribuir de forma significativa a la variabilidad intraespecífica en el peso de semillas. Entre ellos posiblemente el más relevante es el compromiso (trade-off) entre el número y el tamaño (cantidad y calidad) de las semillas. A más producción de semillas es esperable menos calidad de las mismas, ya que los recursos de los que dispone la planta madre para la reproducción hay que repartirlos entre más descendientes potenciales. Este compromiso cantidad-calidad no fue especialmente estudiado en especies arbóreas.

Además de los compromisos entre cantidad y calidad de semilla, existen otros factores que también pueden contribuir a mantener una alta variabilidad intraespecífica en el peso de la semilla, como pueden ser los conflictos paternomaterno en el tamaño óptimo (Willi, 2013), la inestabilidad temporal y espacial en las condiciones ambientales (Charpentier et al., 2012), el hecho de que los recursos limitados reducen la capacidad de las plantas para controlar el tamaño de las semillas (Vaughton y Ramsey, 1998), las variaciones en las presiones selectivas que actúan sobre el peso de la semilla dependiendo de la fase de reclutamiento (Gomez, 2004), o los problemas relacionados con la dispersión y la depredación de las semillas más grandes.

En el proceso de reclutamiento, las semillas tienen que germinar y el tiempo que tardan en germinar puede ser determinante para el éxito de la progenie. Entre otras limitaciones, germinar demasiado pronto puede exponer a la plántula inmadura a lesiones por heladas tardías, mientras que retrasar la germinación puede reducir la capacidad competitiva temprana de la descendencia resultante (Donohue et al., 2010). Valorar la relación entre las características de las semillas y el proceso de germinación resulta, por tanto, de vital importancia para entender las consecuencias finales de los factores que regulan la calidad de la semilla, y en particular, la vecería.

Debido a su estrecha conexión con la planta madre, las semillas están influenciadas no solo por su propio genotipo (House et al., 2010), sino también por el genotipo materno (Schwaegerle y Levin, 1990; Platenkamp y Shaw, 1993; Wolfe, 1995) y por las condiciones ambientales donde creció la planta madre (Roach y Wulff, 1987). Así, las semillas no solo hacen de vehículo para transportar la información genética de los progenitores, sino también de la información no genética sobre el ambiente materno. Estos últimos efectos se conocen como efectos maternos ambientales o plasticidad transgeneracional. Los efectos maternos ambientales pueden transmitirse a la próxima generación a través de diversos mecanismos y pueden tener importantes implicaciones ecológicas y evolutivas. Podría esperarse, por tanto, que la semilla de años veceros dispusiera de mecanismos (reservas, marcas epigenéticas, etc.) que le permita una mayor habilidad competitiva de la progenie resultante. 


\section{Material y Métodos}

\subsection{Material vegetal}

El presente proyecto de investigación se apoya en semillas recogidas en 6 genotipos de Pinus pinaster establecidos en un banco clonal en Boreira (Salcedo, Pontevedra, $42^{\circ} 24^{\prime} 41^{\prime \prime} \mathrm{N}, 08^{\circ} 38^{\prime} 51^{\prime}$ 'O). Los genotipos originales provienen de la región de origen Noroeste Litoral, fueron replicados mediante injerto de la guía principal y se asume que no están emparentados entre sí (Rodríguez, 2019). El banco clonal fue plantado en 2007, incluye varias réplicas clonales de cada genotipo y se estableció con el fin de evaluar la relevancia de los efectos maternos ambientales como fuente de variación fenotípica en $P$. pinaster. Disponemos en total de 25 ramets (réplicas clonales) de 6 genotipos diferentes, con 3 o 6 ramets por genotipo. Desde que los clones entraron en floración (año 2005) se realizó un seguimiento del esfuerzo reproductor de cada árbol en cada año median-

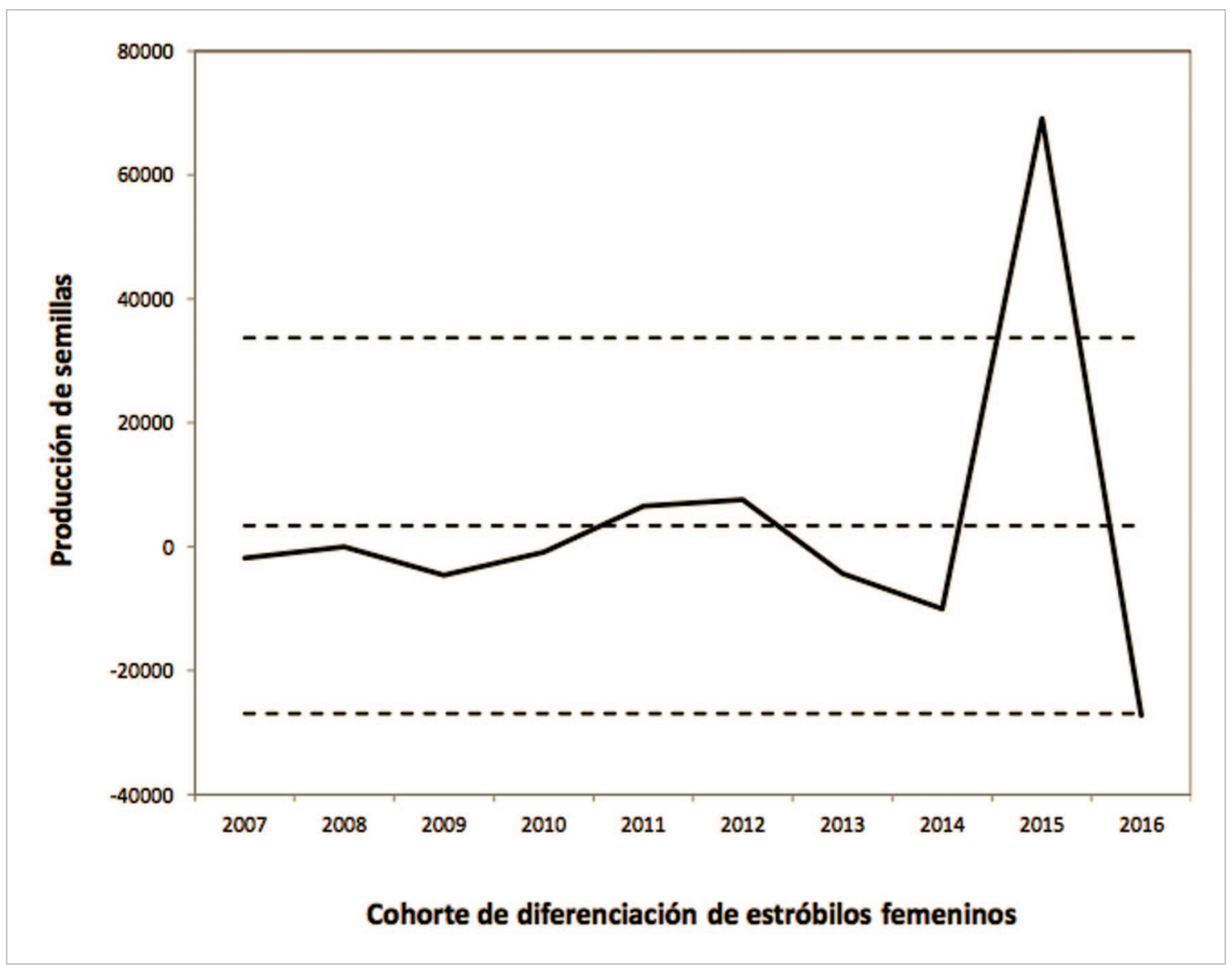

Figura 1. Variación anual de la producción total de semillas sanas. En el eje y se representan los residuos del número de semillas sanas una vez corregidos según el tamaño del árbol. En el eje x se indican los años de diferenciación de estróbilos o cohortes. Las líneas horizontales punteadas indican la desviación típica de la variación anual. La cohorte de 2015 (semillas recogidas en el 2017) se entiende como un año de producción extraordinaria de semillas. 
te el conteo de estróbilos femeninos y masculinos, la recogida de las piñas maduras y la extracción de semillas durante todos los años. Las piñas cosechadas se evaluaron en tamaño y peso tras secado en estufa, extrayendo todas las semillas manualmente. Se disponía, por tanto, de una colección de semillas identificadas de 10 cosechas consecutivas (2007-2017) en los mismos genotipos así como información de la producción (número y peso) de estróbilos, piñas y semillas.

Con esta información se pudo estimar la variación a lo largo de los años del esfuerzo reproductor y el número de semillas viables finalmente producidas en cada año, ajustadas según el incremento en tamaño de los árboles (Figura 1).

Las semillas seleccionadas para este proyecto fueron un total de 2157 . Se analizaron los efectos de la dinámica reproductiva (vecería) en la calidad de la semilla, la germinación y el comportamiento temprano de la progenie. Tres de las 25 semillas seleccionadas de cada ramet se utilizaron para análisis de la calidad propia de la semilla (peso, contenido en nutrientes y concentración en defensas), mientras que las semillas restantes (22 por ramet y año) se utilizaron para evaluar los efectos de la dinámica reproductiva y la calidad de la semilla sobre la germinación (tasa y tiempo de germinación) y desarrollo temprano de la progenie (crecimiento en altura, biomasa total y reparto de biomasa) mediante el establecimiento de un ensayo de progenie bajo condiciones controladas en invernadero.

\section{Resultados}

\section{Efecto de la dinámica reproductiva en la calidad de la semilla}

Las 2157 semillas analizadas tuvieron una biomasa muy variable, siendo la semilla más grande $(0.156 \mathrm{~g})$ algo más de 9 veces más pesada que la más pequeña $(0.017 \mathrm{~g})$ (Tabla 1$)$.

Tabla 1. Promedios, desviaciones estándar, mínimos e máximos de las variables analizadas para análisis de la calidad de las semillas.

\begin{tabular}{lccrr}
\hline \multicolumn{1}{c}{ Variable } & N & Media \pm std & Min & Max \\
\hline & 2157 & $0.078 \pm 0.028$ & 0.017 & 0.156 \\
Peso de la semilla (g) & $85^{1}$ & $9.6 \pm 3.4$ & 3.39 & 16.89 \\
Porcentaje de endospermo (\%) & $85^{1}$ & $13.3 \pm 4.4$ & 5.01 & 23.54 \\
Porcentaje de cáscara (\%) & $85^{1}$ & $2.12 \pm 1.07$ & 0.80 & 5.05 \\
Contido de nitrógeno (\%) & $85^{1}$ & $51.27 \pm 5,36$ & 22.92 & 80.06 \\
Contido de carbono (\%) & $85^{1}$ & $5.12 \pm 1.85$ & 2.42 & 9.65 \\
Concentración de polifenoles $\left(\mathrm{mg} \mathrm{g}^{-1}\right)$ & $85^{1}$ & No detectados & & \\
Concentación de taninos (mg g $\left.{ }^{-1}\right)$ & $85^{1}$ & No detectados & & \\
Concentración de terpenos $\left(\mathrm{mg} \mathrm{g}^{-1}\right)$ & \multicolumn{3}{c}{} \\
\hline
\end{tabular}

${ }^{1}$ Muestras compuestas 
La viabilidad de las semillas fue mayor en los primeros años y menor en los últimos años, incluyendo el año vecero 2015 (Figura 2 a). En el caso del peso de la semilla, se observa una tendencia a pesos más bajos a medida que avanzan los años, siendo el año vecero el de tamaño de semilla más bajo (Figura 2 b). La variación entre cohortes en el porcentaje de endospermo se debe a un anormalmente alto porcentaje de endospermo en la cohorte de 2012 (cogida de 2014) (Figura 2 c). En cuanto el contenido de nitrógeno, los dos últimos años (incluyendo el año vecero 2015) presentan una concentración notablemente inferior (Figura 2 d).

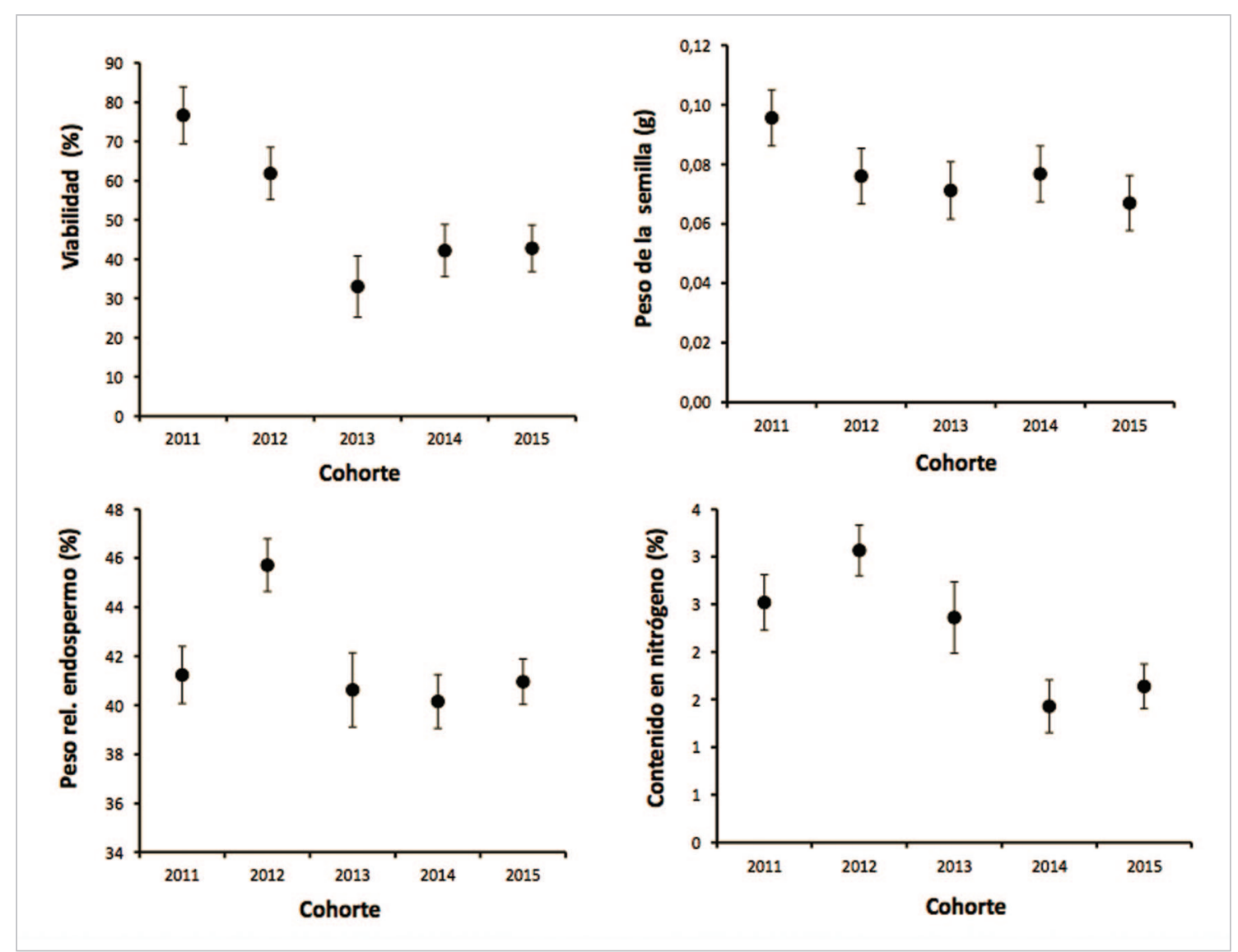

Figura 2. Variación de la calidad de semilla según las cohortes de diferenciación de los estróbilos femeninos a) viabilidad (\% de semillas sanas), b) peso de la semilla $(\mathrm{g})$, c) peso de endospermo (\% respecto al peso total de la semilla) y d) contenido en nitrógeno en el endospermo (\%).

Analizando si la variación interanual en las propiedades de las semillas están relacionadas con el esfuerzo reproductor de los árboles madre, observamos que para todas las variables analizadas, no se detectaron covariación significativa con la producción de semillas de cada árbol en cada año, ni interacción de la variación entre años con los distintos genotipos (Tabla 2, izquierda). 
Tabla 2. Resumen de los modelos mixtos mostrando los resultados del análisis de las dos hipótesis de trabajo para las variables relacionadas con la calidad de la semilla. La primera hipótesis (H1) analiza si la variación interanual en la variable dependiente depende de la producción de semillas sanas de cada árbol madre en cada cohorte. La segunda hipótesis analiza si existe covariación en la variable dependiente con la producción global de todos los árboles de la población maternal en cada cohorte. En ambos casos, se muestra el efecto de la covariable y su interacción con el genotipo. Se muestran los F ratios y el nivel de probabilidad asociada. Los efectos significativos $(\mathrm{p}<0.05)$ se resaltan en negrita.

\begin{tabular}{|c|c|c|c|c|c|c|c|c|c|}
\hline \multirow[b]{3}{*}{ Variable } & \multicolumn{4}{|c|}{$\begin{array}{l}\text { H1: covariación con la producción } \\
\text { de cada rameten cada año }\end{array}$} & \multicolumn{5}{|c|}{$\begin{array}{l}\text { H2: covariacion con la producción } \\
\text { de la población en cada año }\end{array}$} \\
\hline & \multicolumn{2}{|c|}{$\mathbf{N}^{0}$ semillas $^{1}$} & \multicolumn{2}{|c|}{$\mathbf{N}^{0}$ semillas $^{1 *}$ Genotipo } & \multicolumn{2}{|c|}{$\mathrm{N}^{\mathbf{o}}$ semillas $^{2}$} & \multicolumn{3}{|c|}{$\mathrm{N}^{\circ}$ semillas $^{2 *}$ Genotipo } \\
\hline & $\mathbf{F}_{1,63.2}$ & $\mathbf{p}>\mathbf{F}$ & $\mathbf{F}_{5,60.5}$ & $\mathbf{p}>\mathbf{F}$ & $\mathbf{F}^{1,52.4}$ & $\mathbf{p}>\mathbf{F}$ & Efecto & $\mathbf{F}_{5,52.6}$ & $\mathbf{p}>\mathbf{F}$ \\
\hline Peso de semilla & 0,2 & 0,685 & 0,5 & 0,782 & 10,0 & 0,003 & $(-)$ & 0,3 & 0,898 \\
\hline Variabilidad peso & 0,7 & 0,411 & 1,3 & 0,287 & 4,5 & 0,038 & $(+)$ & 0,7 & 0,658 \\
\hline Viabilidad & 3,0 & 0,089 & 1,2 & 0,303 & 2,4 & 0,129 & & 0,3 & 0,900 \\
\hline Endospermo: cáscara & 0,7 & 0,407 & 0,4 & 0,880 & 0,3 & 0,583 & & 0,2 & 0,967 \\
\hline Nitrógeno & 1,0 & 0,334 & 0,9 & 0,479 & 2,0 & 0,160 & & 1,6 & 0,171 \\
\hline $\begin{array}{l}\text { Carbono } \\
\text { Fenoles }\end{array}$ & \multicolumn{9}{|c|}{ No hay variación entre años por lo que no procede el análisis } \\
\hline
\end{tabular}

${ }^{1}$ Producción de semillas sanas en el árbol

${ }^{2}$ Producción de semillas sanas en la población

\section{Efecto de la dinámica reproductiva en la germinación y desarrollo temprano de la progenie}

De las 1903 semillas sembradas germinaron un promedio del 86,9\% (Tabla 3) encontrándose el máximo de germinación en el año 2012 con un $95.16 \%$. El tiempo de germinación varió desde 19 días hasta 136 teniendo un promedio de 59,5 días para todas las semillas (Tabla 3). Las alturas totales de las plántulas variaron entre 0,5 y $5,4 \mathrm{~cm}$ mientras que la biomasa total de las plántulas germinadas varió entre 7 y $583 \mathrm{mg}$. El root-shoot-ratio varió entre 0.24 y 1.51 . El número de raíces laterales fue muy variable, con un mínimo de 0 a un máximo de 131 (Tabla 3).

Tabla 3. Promedios, desviaciones estándar, mínimos e máximos de las variables analizadas para germinación y desarrollo temprano de la progenie.

\begin{tabular}{lcccc}
\hline \multicolumn{1}{c}{ Variable } & N & Media \pm std & Min & Máx \\
\hline Germinación (\%) & & & & \\
Tiempo de germinación (días) & 1903 & $86.93 \pm 61.40$ & 72.41 & 95.16 \\
Altura total (cm) & 1903 & $59.5 \pm 82.7$ & 19 & 136 \\
Biomasa total (g) & 1903 & $6.41 \pm 1.60$ & 0.50 & 5.40 \\
Root: shoot ratio (RSR, 0/1) & 1903 & $0.252 \pm 0.116$ & 0.007 & 0.583 \\
Número de raíces laterales & 1903 & $0.689 \pm 0.180$ & 0.244 & 1.508 \\
& 1903 & $29 \pm 3$ & 0 & 131 \\
\hline
\end{tabular}


Existe un claro descenso de la tasa de germinación en el año vecero 2015 a diferencia de los otros años no veceros (Figura 3 a), como también sucede con el tiempo de germinación (Figura 3 b). La relación en las alturas totales a lo largo de los años tiene distinción significativa entre años, observando como el año vecero 2015 genera plántulas de ligera menor altura (Figura 3 c). Referente a la biomasa total de las plántulas, se nota un ligero descenso en los últimos años, incluyendo el año vecero 2015 (Figura 3 d).

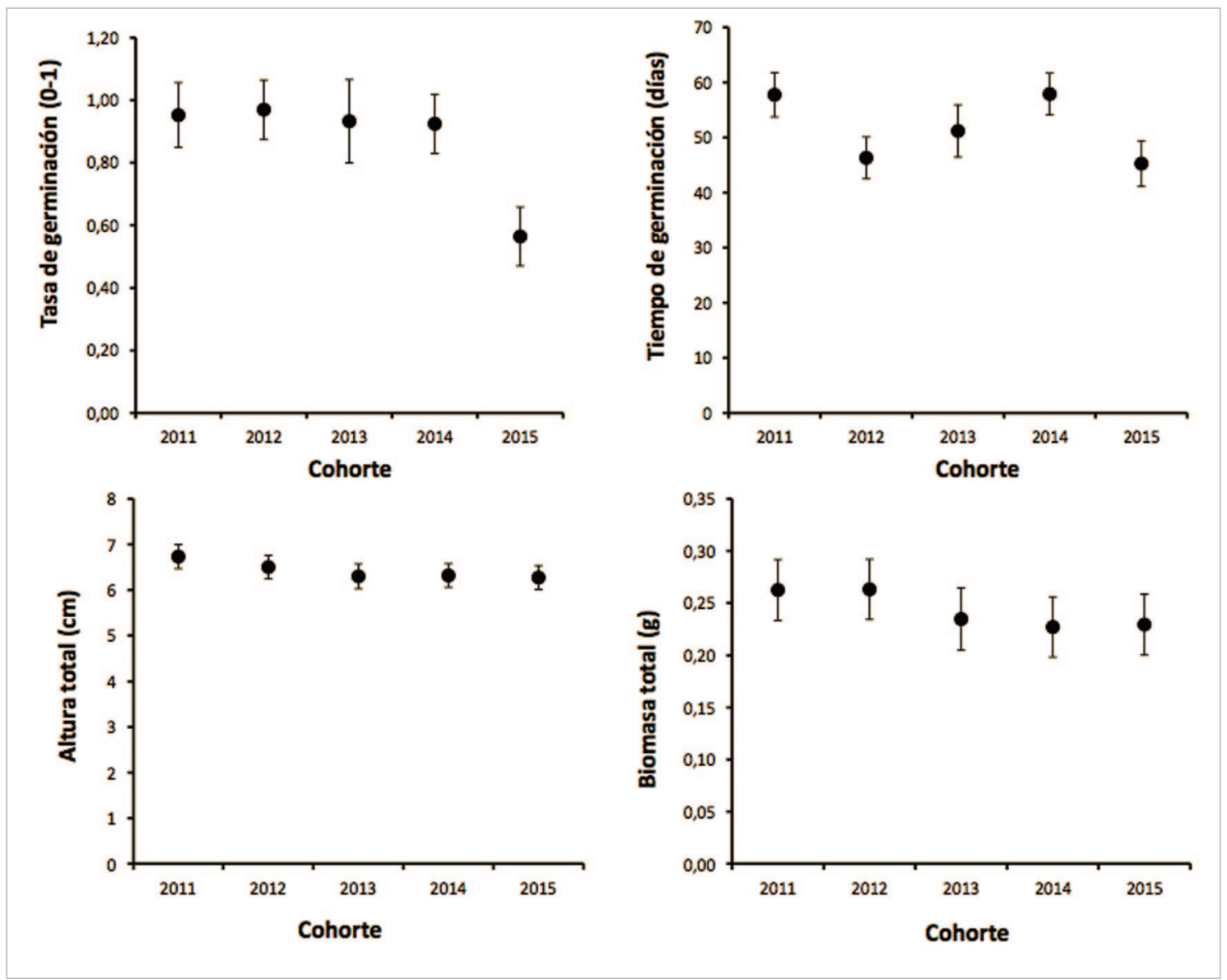

Figura 3. Variación de la germinación y desarrollo temprano en función de la cohorte de diferenciación de estróbilos femeninos a) tasa de germinación, b) tiempo de germinación (días), c) altura total de las plántulas $(\mathrm{cm})$ y d) biomasa total de las plántulas tras cuatro meses desde la siembra $(\mathrm{g})$.

La tasa de germinación varió tanto entre cohortes como entre genotipos maternos. En la figura 4 se aprecia que esta interacción se debe la una anormalmente baja tasa de germinación de tres genotipos maternos en el año vecero.

En cuanto a las diferencias entre invernaderos, las plantas del invernadero 2 germinaron ligeramente mejor (Figura 5 a) y un poco antes (Figura 5 b) pero consiguieron una altura (Figura 5 c) y una biomasa total (Figura 5 d) ligeramente menor que las plantas del invernadero 1. 


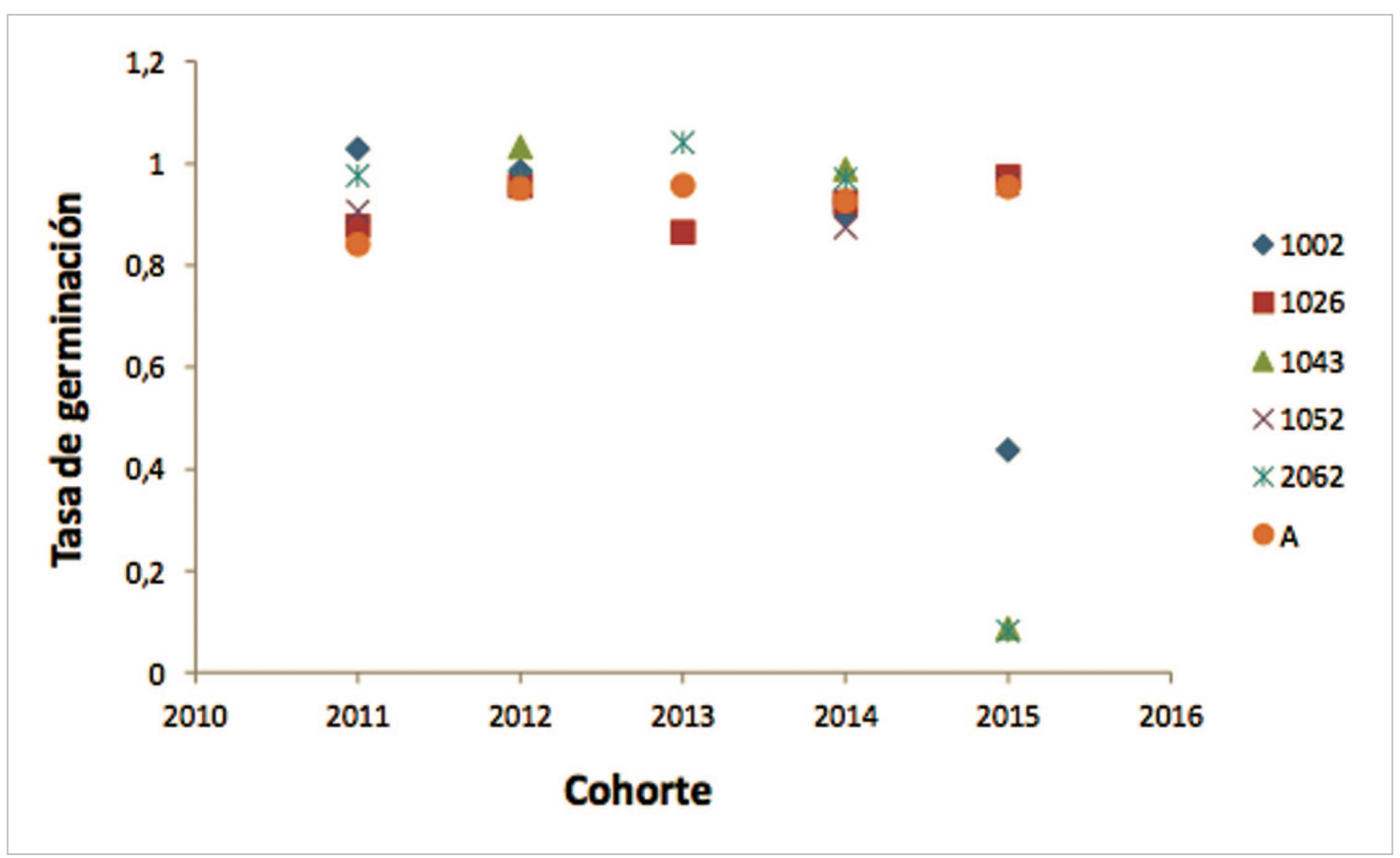

Figura 4. Interacción cohorte $\mathrm{x}$ genotipo para la tasa de germinación mostrando la tasa de germinación de la semilla producida en los distintos años para cada genotipo materno.

Observando la interacción del genotipo con la producción de la población encontramos significación sobre la variable de germinación (Tabla 6, derecha), lo que indica que la covariación de esta variable con la producción de semillas sanas de la población fue variable entre genotipos (Tabla 6, derecha).

\section{Discusión}

El peso de la semilla no solo fue controlado por el genotipo materno sino también por efectos ambientales. Basándonos en las observaciones de la variación del peso de la semilla entre cohortes ésta fue fuertemente significativa. Esta variación interanual en el peso de la semilla puede darse por numerosos factores, tanto efectos ambientales asociados a la variabilidad interanual en las condiciones climáticas, como aquellos que emanan del posible compromiso entre número y tamaño de semillas producidas (Sadras, 2007). Esta idea de un compromiso entre la cantidad y calidad de las semillas producidas constituye nuestra primera hipótesis de trabajo y se justifica por la necesidad de repartir los recursos disponibles (normalmente escasos) entre el conjunto de las semillas producidas por cada árbol en cada año. Evaluando esta hipótesis (Tabla 2, izquierda) se comprobó que la variación interanual en el peso de la semilla no depende del esfuerzo reproductor de cada árbol en cada año. No parece, por tanto, que el mayor o menor peso de las semillas en los distintos años tenga que ver con conflictos calidad-cantidad en el reparto de recursos. No 

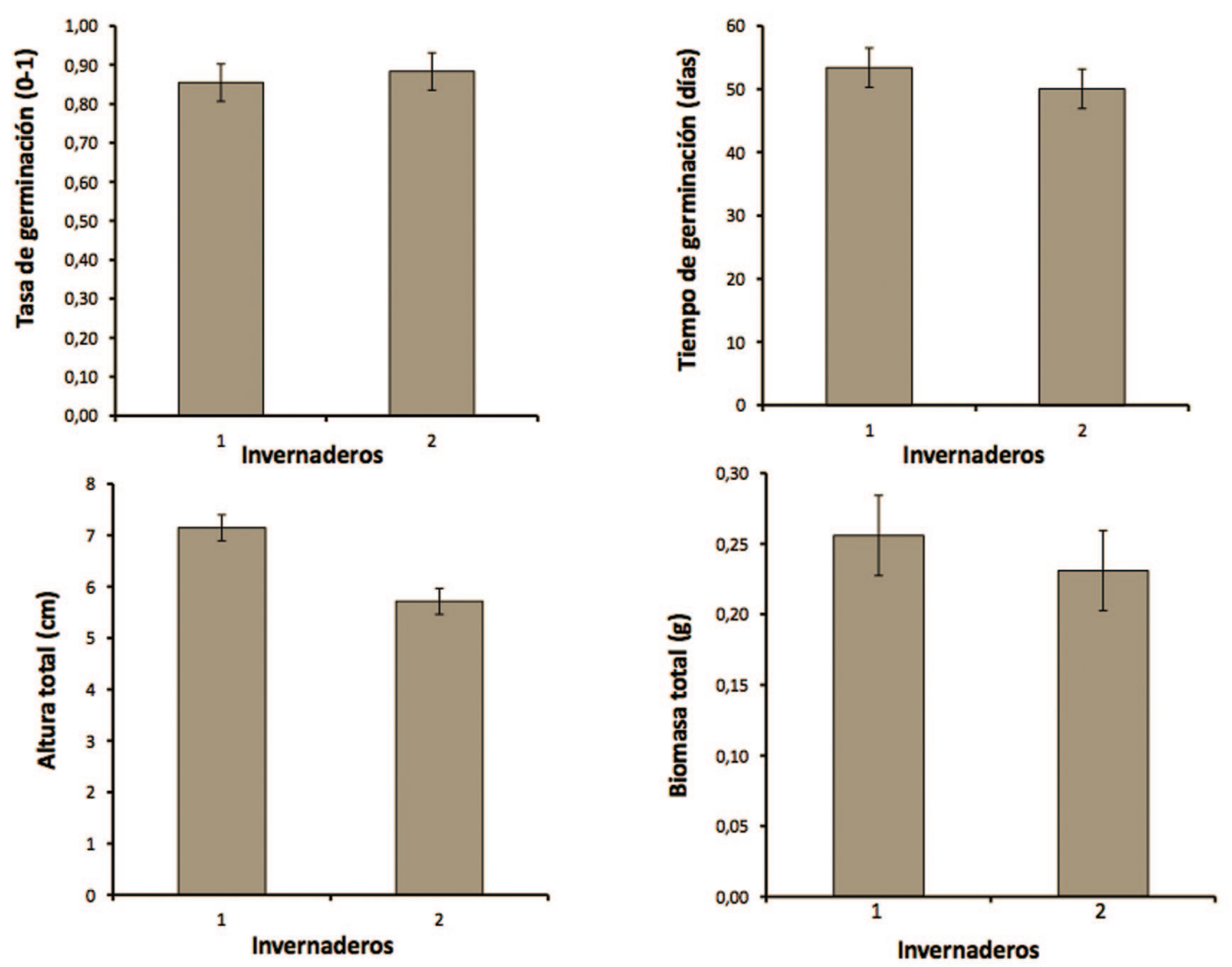

Figura 5. Diferencias entre el invernadoiro 1 y 2 en la a) tasa de germinación, b) tiempo de germinación (días), c) altura total (cm) e d) biomasa total (g).

Tabla 4. Resumen de los modelos mixtos mostrando los resultados del análisis de las dos hipoteses de trabajo para las variables relacionadas con la germinación y desarrollo temprano de la progenie. La primera hipótesis (H1) analiza si la variación interanual en la variable dependiente depende de la producción de semillas sanas de cada árbol madre en cada cohorte. La segunda hipótesis analiza se existe covariación en la variable dependiente con la producción global de todos los árboles de la población maternal en cada cohorte. En ambos casos, se muestra el efecto de la covariable y su interacción con el genotipo. Se muestran los F ratios y el nivel de probabilidad asociada. Los efectos significativos $(p<0.05)$ se resaltan en negrita.

\begin{tabular}{|c|c|c|c|c|c|c|c|c|c|c|}
\hline & \multicolumn{5}{|c|}{$\begin{array}{l}\text { H1: covariación con la producción } \\
\text { de cada rameten cada año }\end{array}$} & \multicolumn{5}{|c|}{$\begin{array}{l}\text { H2: covariacion con la producción } \\
\text { de la población en cada año }\end{array}$} \\
\hline & \multicolumn{2}{|c|}{$\mathbf{N}^{0}$ semillas $^{1}$} & \multicolumn{3}{|c|}{$\mathrm{N}^{\circ}$ semillas ${ }^{1 *}$ Genotipo } & \multicolumn{2}{|c|}{$\mathbf{N}^{0}$ semillas $^{2}$} & \multicolumn{3}{|c|}{$\mathrm{N}^{0}$ semillas $^{2 *}$ Genotipo } \\
\hline Variable & $\mathbf{F}^{1,632}$ & $\mathbf{p}>\mathbf{F}$ & Efecto & $\mathbf{F}_{5,60.5}$ & $\mathbf{p}>\mathbf{F}$ & $\mathbf{F}^{1,63.2}$ & $\mathbf{p}>\mathbf{F}$ & Efecto & $\mathbf{F}_{5,60.5}$ & $\mathbf{p}>\mathbf{F}$ \\
\hline Germinación & 0,6 & 0,439 & & 0,2 & 0,973 & 229,7 & $<0.001$ & $(+)$ & 74,2 & $<0.001$ \\
\hline Tiempo germinación & 4,3 & 0,043 & $(+)$ & 1,9 & 0,104 & 6,4 & 0,013 & $(-)$ & 1,2 & 0,334 \\
\hline Htotal & 3,3 & 0,077 & & 1,5 & 0,212 & 6,8 & 0,010 & $(-)$ & 2,3 & 0,050 \\
\hline TDW & 3,0 & 0,090 & & 1,2 & 0,339 & 4,9 & 0,032 & $(+)$ & 0,9 & 0,495 \\
\hline $\begin{array}{l}\text { RSR } \\
\text { Nroots }\end{array}$ & \multicolumn{10}{|c|}{ No hay variación entre años por lo que no procede el análisis } \\
\hline
\end{tabular}

${ }^{1}$ Producción de semillas sanas en el árbol. ${ }^{2}$ Producción de semillas sanas en la población. 
obstante, la variación interanual del peso de la semilla covarió notablemente con la producción global de semillas de la población en cada año (Tabla 2, derecha), con un estimador de efecto negativo que indica que a mayor producción de semillas por la población más pequeñas son las semillas (años veceros semillas más pequeñas). De esta manera, la semilla de años veceros es diferente a la de años no veceros, pero estas diferencias no se deben a compromisos por los recursos a nivel de árbol individual, sino a un efecto poblacional, en el que el tamaño de la semilla depende directamente del esfuerzo reproductor de toda la población. La vecería parece jugar un papel prioritario en la decisión del tamaño de las semillas, aunque es difícil explicar qué posibles ventajas adaptativas pueden tener las semillas más pequeñas producidas en los años de producción extraordinaria de semillas.

Teniendo en cuenta la heterogeneidad ambiental en las que se establecen las semillas (Valladares et al., 2007), la variabilidad en el peso de la semilla puede dar ventaja para enfrentar los compromisos entre depredación, dispersión y desarrollo temprano de la plántula. Una mayor variabilidad en el tamaño de la semilla en años de máxima producción de semillas puede ser la mejor estrategia para que, al menos un porcentaje de las semillas producidas, germine y contribuya el reclutamiento de la población.

Es importante señalar que la inversión de recursos destinados a defensas químicas en las semillas no son muchos. Los análisis químicos no permitieron encontrar, ni taninos ni mono y sesquiterpenos, siendo la concentración de polifenois totales muy baja y claramente inferior a observada comúnmente en tejidos de pino (Sampedro et al., 2011). Pero sí que existe inversión de recursos para reservas, de hecho, casi todos los recursos se invierten en reservas. En nuestro estudio aparece mucho contenido en carbono (Tabla 1) así como muchos ácidos grasos que se encontraron en los análisis cromatográficos pero que no fue posible identificar ni cuantificar. Los resultados también mostraron una fuerte variación en la tasa de germinación a lo largo de los años, pero la variación interanual no fue igual sino que depende del genotipo (Tabla 3).

La tasa de germinación fue mucho menor en semillas procedentes de años veceros que en semillas de años no veceros, siendo este efecto marcado por una falta casi absoluta de germinación de la semilla en el año 2015 (año de máxima producción de semillas) de ciertos genotipos. La escasa germinación de la semilla del año vecero de algunos genotipos podría ser resultado también de los compromisos entre producir mucho y producir semillas que sean válidas. No obstante, los resultados indican que el esfuerzo reproductor de cada árbol no covarió con la tasa de germinación (Tabla 4, izquierda) sugiriendo que estos compromisos no son los responsables. Aunque no podemos concluir sobre las causas de este marcado efecto de la vecería sobre la tasa de germinación, este efecto coincide con nuestra segunda hipótesis y sugiere que producir muchas semillas a nivel poblacional tiene consecuencias sobre las características de las semillas producidas, en particular sobre la viabilidad de las mismas y por tanto sobre la eficacia biológica. El tiempo de germinación para el año vecero 2015 fue claramente menor que en los otros años no veceros. En este caso, la variación anual en el tiempo de germinación covarió significativamente tanto con el esfuerzo reproductor de cada ramet en cada año (Tabla 4, izquierda) como con el esfuerzo reproductor global de la población en su conjunto (Tabla 4, derecha). 
El primero indica que el efecto podría estar mediado por conflictos en el reparto de recursos a nivel de individuo, mientras que el segundo sugiere un cambio en las propiedades de la semilla en función de características de la población. Este último efecto podría relacionarse con efectos maternos ambientales tal como muestran los trabajos de Cendán et al. (2013) donde las condiciones ambientales donde se desarrolla la madre determinan el tiempo de germinación de la semilla. Así, en un año vecero, las plantas madre podrían adelantar el tiempo de germinación para facilitar el desarrollo de la progenie en un ambiente donde es previsible una mayor densidad de reclutamiento y por lo tanto de competencia.

Se sabe que el peso de la semilla es un carácter de mucha importancia evolutiva que está bastante influido por el entorno materno y que tiene importantes consecuencias en la germinación y desarrollo temprano de la progenie (Linkies et al., 2010). Los resultados del presente TFG confirman el anterior, observándose covariación del peso de la semilla con la mayoría de las variables relacionadas con la germinación y desarrollo temprano de la progenie.

El peso de la semilla tuvo una covariación importante influyendo directamente en la tasa y tiempo de germinación así como en el crecimiento inicial de la progenie. Los signos de los estimadores de esta covariación indican que, cuanto más peso de semilla mayor tasa de germinación, antes germinan las semillas y mayor crecimiento inicial de la progenie. Estos resultados coinciden con resultados previos (Cendán et al.., 2013; Zas et al., 2013).

Es importante señalar que la mayoría de los caracteres estudiados relacionados con la calidad de la semilla, la germinación y el desarrollo temprano de la progenie mostraron una fuerte variación genética (Tabla 3), algo que estudios anteriores ya mencionaron (Wu et al., 2008). Los patrones de variación temporal y su dependencia del esfuerzo reproductor de los árboles o de la población en su conjunto son comunes para todos los genotipos, sugiriendo que son patrones sincronizados entre los distintos genotipos de una población, una de las características básicas de la vecería como estrategia reproductiva (Rodríguez, 2019).

Teniendo en cuenta los resultados en su conjunto, observamos que existe una fuerte variación interanual en la mayoria de los caracteres analizados tanto en relación con la calidad de la semilla como con la germinación y el desarrollo temprano de las plántulas. En general, esta variación anual no está relacionada con el esfuerzo reproductor de cada individuo, por lo que nuestra hipótesis de que las semillas de años veceros son de peor calidad por conflictos en el reparto de recursos (compromisos entre cantidad y calidad) a nivel de individuo tiene que ser descartada. No obstante, la mayoría de los caracteres muestran un efecto de la vecería a nivel poblacional, donde los distintos caracteres dependen de la producción de semillas de la población en su conjunto. Se observó un efecto poblacional de la vecería sobre el peso de la semilla, la variabilidad intraárbol en el peso de la semilla, la tasa de germinación, el tiempo de germinación y el crecimiento y la biomasa de las plántulas. A más producción de semillas en el conjunto de la población, menor peso de semi1la, mayor variabilidad en el peso de la semilla, menor tasa de germinación y germinación adelantada, y mayor crecimiento y biomasa de las plántulas. 


\section{Conclusiones}

1. Se observó una fuerte variación entre años en la mayoría das variables relacionadas con la calidad de la semilla, la germinación y el desarrollo temprano de la progenie.

2. Las plántulas resultantes de las semillas del año vecero crecieron ligeramente menos y acumularon menos biomasa que las procedentes de semillas de años normales.

3. La variación anual en los distintos caracteres no dependen de conflictos en el reparto de recursos entre las semillas producidas a nivel de individuo materno.

4. La vecería condiciona la calidad de la semilla, germinación y desarrollo temprano de la progenie a nivel poblacional.

5. Predisposición de las semillas de años veceros para desarrollarse en un ambiente de mayor densidad de plántulas y, por lo tanto, más competitivo.

\section{Agradecimientos}

A todas las personas del departamento de Ecología Forestal del CSIC de Pontevedra, en especial a Rafa, Asier y Luis. A mi familia, amigas/amigos, a mi mozo y a Polka, mi gran compañera de vida.

\section{Bibliografía}

Avedaño, María de la luz; 2016. La reprodución de las plantas: costos y beneficios. Ciencia, octubre-diciembre.

Cendán, C.; Sampedro, L.; Zas, R.; 2013. The maternal environment determines the timing of germination in Pinus pinaster. Environmental and Experimental Botany 94, 66-72. https://doi.org/10.1016/j.envexpbot.2011.11.022

Charpentier, A.; Anand, A.; Bauch, C.T.; 2012. Variable offspring size as an adaptation to environmental heterogeneity in a clonal plant species: integrating experimental and modelling approaches. Journal of Ecology 100, 184-195. https://doi.org/10.1111/j.1365-2745.20 11.01899.x

Donohue, K.; Rubio de Casas, R.; Burghardt, L.; Kovach, K.; Willis, C.G.; 2010. Germination, postgermination adaptation, and species ecological ranges. The Annual Review of Ecology, Evolution, and Systematics 41, 293-319. https://doi.org/10.1146/annurev-ecolsys-102209144715

Gómez, J.M.; 2004. Bigger is not always better: conflicting selective pressures on seed size in Quercus ilex. Evolution 58, 71-80. https://doi.org/10.1111/j.0014-3820.2004.tb01574.x 
House, C.; Roth, C.; Hunt, J.; Kover, P.X.; 2010. Paternal effects in Arabidopsis indicate that offspring can influence their own size. Proceedings of the Royal Society B-Biological Sciences 277, 2885-2893. https://doi.org/10.1098/rspb.2010.0572

Roach, D.A.; Wulff, R.D.; 1987. Maternal effects in plants. Annual Review of Ecology and Systematics 18, 209-235. https://doi.org/10.1146/annurev.es.18.110187.001233

Ramirez-Valiente, J.A.; Valladares, F.; Gil, L.; Aranda, I.; 2009. Population differences in juvenile survival under increasing drought are mediated by seed size in cork oak (Quercus suber L.). Forest Ecology and Management 257, 1676-1683. https://doi.org/10.1016/j.foreco.2009.01.024

Rodríguez, S.; 2019. Efecto de la dinámica reproductiva en la habilidad competitiva de la progenie de Pinus pinaster. Trabajo Fin de Grado. Grado de Biologia, Universidad de Vigo, 27 pp.

Sadras, V.O.; 2007. Evolutionary aspects of the trade-off between seed size and number in crops. Field Crops Research 100, 125-138. https://doi.org/10.1016/j.fcr.2006.07.004

Santos-del-Blanco, L.; Climent, J.; Bonser, S.; 2014. Costs of female reproduction in a conifer tree: a whole-tree level assessment. Journal of Ecology 102, 1310-1317. https://doi.org/10.1 $111 / 1365-2745.12283$

Schwaegerle, K.E.; Levin, D.A.; 1990. Environmental-Effects on growth and fruit production in phlox-drummondii. Journal of Ecology 78, 15-26. https://doi.org/10.2307/2261033

Vaughton, G.; Ramsey, M.; 1998. Sources and consequences of seed mass variation in Banksia marginata (Proteaceae). Journal of Ecology 86, 563-573. https://doi.org/10.1046/j.1365-27 45.1998.00279.x

Wolff, J.O.; 1996. Population fluctuations of mast-eating rodents are correlated with production of acorns. J Mamma. 77, 850-856. https://doi.org/10.2307/1382690

Willi, Y.; 2013. The battle of the sexes over seed size: Support for both kinship genomic imprinting and interlocus contest evolution. American Naturalist 181, 787-798. https://doi.org/ $10.1086 / 670196$

Zas, R.; Sampedro, L.; 2015. Heritability of seed weight in Maritime pine, a relevant trait in the transmission of environmental maternal effects. Heredity 114, 116-124. https://doi.org/ 10.1038/hdy.2014.76

Zas, R.; Cendan, C.; Sampedro, L.; 2013. Mediation of seed provisioning in the transmission of environmental maternal effects in Maritime pine (Pinus pinaster Aiton). Heredity 111, 248255. https://doi.org/10.1038/hdy.2013.44 
